Integr. equ. oper. theory 35 (1999) $1-19$

$0378-620 \mathrm{X} / 99 / 010001-19 \$ 1.50+0.20 / 0$

(C) Birkhäuser Verlag, Basel, 1999

\title{
BOUNDED AND COMPACT MULTIPLIERS BETWEEN BERGMAN AND HARDY SPACES
}

\author{
STEPHEN M. BUCKLEY, M. S. RAMANUJAN, AND DRAGAN VUKOTIĆ
}

This paper studies the boundedness and compactness of the coefficient multiplier operators between various Bergman spaces $A^{p}$ and Hardy spaces $H^{q}$. Some new characterizations of the multipliers between the spaces with exponents 1 or 2 are derived which, in particular, imply a Bergman space analogue of the Paley-Rudin Theorem on sparse sequences. Hardy and Bergman spaces are shown to be linked using mixed-norm spaces, and this linkage is used to improve a known result on $\left(A^{p}, A^{2}\right), 1<p<2$.

Compact $\left(H^{1}, H^{2}\right)$ and $\left(A^{1}, A^{2}\right)$ multipliers are characterized. The essential norms and spectra of some multiplier operators are computed. It is shown that for $p>1$ there exist bounded non-compact multiplier operators from $A^{p}$ to $A^{q}$ if and only if $p \leq q$.

\section{INTRODUCTION}

The coefficients of, and multipliers between, various function spaces is a rather old and wellstudied subject that has seen a recent revival of interest. Some ideas and techniques appeared only in the last few years, and there are still many natural questions to be answered. In this paper we attempt to tie in old and new results, in order to draw several conclusions not found elsewhere. We discuss both boundedness and compactness of multiplier operators; the latter topic appears to have been neglected in the literature.

The history of multipliers on function spaces is rather long, so we shall content ourselves here with a brief review of it. The subject finds its real beginnings in the classical theorems of Hardy, Littlewood, and Paley (cf. [20], [28], [12]) on integration, differentiation, and order of growth of analytic functions in the disk. The spaces $H(p, q, \alpha)$ (of which both $A^{p}$ and $H^{p}$ are special cases) had already been studied by Hardy and Littlewood but were explicitly defined and investigated by Flett (cf. [17], [18], [19]), and in certain cases by Duren-Shields ([14], [15]), Sledd [37], Ahern-Jevtić [1], and others. Some precise results on the multipliers of Hardy spaces were obtained by Duren [13], Hedlund [22], Sledd ([38], [39]), Hare [21], Mateljević-Pavlović [31], Jevtić-Jovanović [24], etc.; see also [11].

Results on coefficients of functions in various function spaces can be found, for 
example, in the articles by Flett [17], Duren-Taylor [16], Horowitz [23], Nakamura-OhyaWatanabe [32], Mateljević-Pavlović [30], Blasco [7], and Buckley-Koskela-Vukotić [8].

Pavlović [33] characterized $\left(A_{\alpha}^{p}, A_{\alpha}^{q}\right)$ for $0<p \leq 1, p \leq q<\infty$, and $\alpha>-1$ in terms of the growth of the integral means of an associated analytic function; Lou [29] obtained a similar condition for $\left(A^{1}, A^{q}\right), q \geq 1$. The conditions are, however, difficult to verify in practice, as we cannot easily control the growth of a function in terms of its coefficients if $p \neq 2$. It is therefore interesting, even in these cases, to determine (usually separate) necessary and sufficient conditions for membership in multiplier spaces in terms of concrete sequence spaces.

Wojtaszczyk made a breakthrough by characterizing $\left(A^{p}, A^{q}\right)$ when $q \leq 2 \leq p$ in terms of mixed-norm spaces (see Theorem B below). [40] and [8] give simple, but separate, necessary and sufficient conditions for membership in all other cases; in addition, [40] gives a characterization of $\left(A^{1}, A^{2}\right)$ analogous to that of $\left(H^{1}, H^{2}\right)$ obtained by Hardy and Littlewood.

Blasco [7] extended several results from [30], [42], and [40]. For example, he characterized $\left(A^{p}, A^{2}\right)$ for $p \in(0,1]$, and described $\left(A^{p}, A^{q}\right)$ for $0<p, q \leq 1$ in terms of certain related integral means. Also his earlier paper [6] gives a very general and useful result about bounded operators on $A^{1}$.

This paper is a continuation of the articles [40] and [8] with a different focus: we are also interested in the compactness of coefficient multipliplier operators. In Section 2, we first review some basic definitions and facts. In Section 3 we obtain some new conclusions about the spaces $H^{p}$ and $A^{p}$ for $p=1,2$; in particular, we get a version of the PaleyRudin Theorem for Bergman spaces. In Section 4 we investigate compactness of multiplier operators. We compute the essential norms and spectra of some multiplier operators and prove the compact versions of some results for spaces with indices 1,2 . We also show that for $p>1$ the bounded and compact $\left(A^{p}, A^{q}\right)$ multipliers coincide precisely when $q<p$. In Section 5 we mention some open questions.

In order to avoid confusing new with old results, many of which are stated in the paper, we partition them into two groups. The theorems which were obtained earlier (whether by some of us or by other authors) are denoted by capital letters and given without proof, while our own theorems (as well as the new observations obtained by combining earlier results) are enumerated.

\section{NotATION AND BACKGROUND}

Throughout the text, $\mathbf{D}=\{|z|<1\}$ will denote the unit disc in the complex plane. $d A(z)=$ $\frac{1}{\pi} d x d y(z=x+i y)$ is the normalized Lebesgue area measure on $\mathbf{D} . \mathcal{H}(\mathbf{D})$ is the algebra of holomorphic functions in D. $X, Y$ will always be used to denote spaces of analytic functions on $\mathrm{D}$ satisfying certain integrability conditions (such as $H^{p}$ and $A_{\alpha}^{p}$ spaces). For $f \in \mathcal{H}(\mathrm{D})$, $0<r<1$, the integral means $M_{p}(r, f)$ are defined by

$$
M_{p}(r, f)=\left(\frac{1}{2 \pi} \int_{0}^{2 \pi}\left|f\left(r e^{i \theta}\right)\right|^{p} d \theta\right)^{1 / p}
$$


and are increasing with $r$. The Hardy space $H^{p}(0<p<\infty)$ is the space of all $f \in \mathcal{H}(\mathrm{D})$ for which $\|f\|_{H^{p}}=\lim _{r \rightarrow 1} M_{p}(r, f)<\infty$, and $H^{\infty}$ is the space of bounded $f \in \mathcal{H}(\mathrm{D})$. The weighted Hardy spaces $H_{\alpha}^{p}$ consist of all $f \in \mathcal{H}(\mathrm{D})$ such that

$$
\sup _{0<r<1}(1-r)^{\alpha} M_{p}(r, f)<\infty \text {. }
$$

The space $H(p, q, s), 0<p \leq \infty, 0<q, s<\infty$ consists of all $f \in \mathcal{H}(\mathbf{D})$ for which

$$
\|f\|_{H(p, q, s)} \equiv\left(\int_{0}^{1} M_{p}(r, f)^{q}(1-r)^{s q-1} d r\right)^{1 / q}<\infty
$$

The weighted Bergman space $A_{\alpha}^{p}$ is defined by $A_{\alpha}^{p}=H(p, p,(1+\alpha) / p)$ for $\alpha>-1$. Equivalently, $A_{\alpha}^{p}$ is the set of all $f \in \mathcal{H}(\mathbf{D})$ for which

$$
\|f\|_{A_{\alpha}^{p}}=\left\{\int_{D}|f(z)|^{p}\left(1-|z|^{2}\right)^{\alpha} d A(z)\right\}^{1 / p}<\infty
$$

The (unweighted) Bergman space $A^{p}$ is simply $A_{0}^{p}$.

We identify a function $f \in \mathcal{H}(\mathbf{D})$ with its sequence of Taylor coefficients $\left(a_{n}\right)_{n=0}^{\infty}$ (and so, for example, we identify the spaces $H^{2}$ and $l^{2}$ ). Formulae such as $\|f\|_{H^{2}}^{2}=\sum_{n=0}^{\infty}\left|a_{n}\right|^{2}$ and $\|f\|_{A^{2}}^{2}=\sum_{n=0}^{\infty}\left|a_{n}\right|^{2} /(n+1)$ are well-known. For $p \neq 2$, there are summability conditions on the coefficients that are either sufficient or necessary for membership in $H^{p}$ or $A_{\alpha}^{p}$, but they do not coincide in general (see [8], [12], [32], [40]).

For any exponent $1<p<\infty, p^{\prime}$ will always denote the dual exponent $p /(p-1)$. The duality relation $\left(A^{p}\right)^{*}=A^{p^{\prime}}$ holds for $1<p<\infty$ with respect to the pairing

$$
<f, g>=\int_{\mathbf{D}} f \bar{g} d A=\sum_{n=1}^{\infty} a_{n} \overline{b_{n}}(n+1)^{-1},
$$

where, $f \sim\left(a_{n}\right)_{n=0}^{\infty}$ and $g \sim\left(b_{n}\right)_{n=0}^{\infty}$ (see [5] or [44]). Furthermore the $\left(A^{p}\right)^{*}$-norm of the linear functional corresponding to $g \in A^{p^{\prime}}$ is comparable with $\|g\|_{A^{p^{\prime}}}$.

A function $f \in \mathcal{H}(\mathrm{D})$ is said to belong to the Bloch space $\mathcal{B}$ if $\left(1-|z|^{2}\right)\left|f^{\prime}(z)\right|$ is bounded in $\mathbf{D}$. $\mathcal{B}$ is a Banach space when equipped with the norm

$$
\|f\|_{\mathcal{B}}=|f(0)|+\sup _{z \in \mathcal{D}}\left(1-|z|^{2}\right)\left|f^{\prime}(z)\right| \text {. }
$$

If, moreover, $\lim _{|z| \rightarrow 1^{-}}\left(1-|z|^{2}\right)\left|f^{\prime}(z)\right|=0$ holds, we say that $f$ lies in the little Bloch space $\mathcal{B}_{0}$. Useful background on these spaces can be found in [2]. Using the same pairing as above, we have $\mathcal{B}_{0}^{*}=A^{1}$ and $\left(A^{1}\right)^{*}=\mathcal{B}$; see [5], [2], or [44].

Given a sequence $\lambda=\left(\lambda_{n}\right)_{n=0}^{\infty}$ and two function spaces $X, Y$, we define the multiplier operator $T_{\lambda}$ in the natural way: if $f \in X, f(z)=\sum_{n=0}^{\infty} a_{n} z^{n}$, then $\left(T_{\lambda} f\right)(z)=\sum_{n=0}^{\infty} \lambda_{n} a_{n} z^{n}$. If $T_{\lambda}: X \rightarrow Y$, then $\lambda$ is said to be a multiplier from $X$ into $Y$; we denote by $(X, Y)$ the class of all such multipliers.

In what follows we will assume that the point-evaluation functionals on the space $X$ are bounded. Together with the Closed Graph Theorem, this immediately implies that $\lambda=\left(\lambda_{n}\right) \in(X, Y)$ if and only if $T_{\lambda} \in \mathcal{L}(X, Y)$, the space of bounded operators from $X$ 
to $Y$. Note that, although $H^{p}$ and $A^{p}$ are not Banach spaces for $0<p<1$, they do have a complete translation-invariant metric (distance from the origin is the $p$ th power of the "norm"), and so the Closed Graph Theorem is applicable to all $H^{p}$ and $A^{p}$ spaces. We are also interested in investigating when $T_{\lambda} \in \mathcal{K}(X, Y)$, the space of compact operators from $X$ to $Y$.

Some statements about $(X, Y)$ require cumbersome notation if written precisely. We shall replace $n+1$ (wherever it appears) by $n$, ignoring division-by-zero problems. This imprecision is harmless, as it is easily seen that $\left(\lambda_{n+1}\right)_{n=0}^{\infty} \in(X, Y) \Leftrightarrow\left(\lambda_{n}\right)_{n=0}^{\infty} \in(X, Y)$ for any of the spaces of interest to us.

A particularly important family of multipliers is that of the fractional derivatives $D^{t}, t \in \mathbf{R}$ (usually called fractional integral operators if $t<0$ ). We essentially define $D^{t}$ to be the multiplier $\left(n^{t}\right)$, except that we define its coefficient of index zero to be 1 , no matter what the value of $t$ (this variant definition of the initial coefficient simplifies the statements of some later results). For any function space $X$, we write $D^{t} X=\left\{D^{t} f: f \in X\right\}$.

We next wish to define very general mixed norm sequence spaces, but let us begin with some notation. Here and later, it is sometimes convenient to write sequences $\left(a_{n}\right)_{n=0}^{\infty}$ as formal power series $\sum_{n=0}^{\infty} a_{n} x^{n}$ (these series will always involve powers of $x$ to distinguish them from analytic functions which are written as power series in $z$ ). We define $I_{k}$ to be the $k$ th dyadic block of integers, i.e. $I_{k}=\left\{2^{k-1}, \ldots, 2^{k}-1\right\}$ for $k>0$, and $I_{0}=\{0\}$. Finally, on any sequence space, we define the operator $S_{k}, k \geq 0$, which selects out the $k$ th dyadic block of terms and shifts it to an initial position. More precisely, $S_{0}\left(\sum_{n=0}^{\infty} a_{n} x^{n}\right)=a_{0} x^{0}$ and $S_{k}\left(\sum_{n=0}^{\infty} a_{n} x^{n}\right)=\sum_{n \in I_{k}}^{\infty} a_{n} x^{n-2^{k-1}}$.

We are now ready to define our mixed norm spaces. If $A, B$ are sequence spaces, and $B$ has a norm, the new sequence space $A[B]$ consists of all sequences $\lambda$ such that $\left(\left\|S_{k} \lambda\right\|_{B}\right)_{k=0}^{\infty} \in A$. If $A$ is also a normed space, then $A[B]$ is a normed space with

$$
\|\lambda\|_{A[B]}=\left\|\left(\left\|S_{k} \lambda\right\|_{B}\right)_{k=0}^{\infty}\right\|_{A} .
$$

We can iterate this construction to define spaces of the form $A[B[C]]$, and so on.

One could also define $A[B]$ as above, if membership in $B$ is given by the finiteness of a quantity $\|\cdot\|_{B}$ which is not a genuine norm; we do so only for $B=l^{p}, p>0$, in which case $\left\|\left(a_{n}\right)\right\|_{l p}$ is defined, as usual, to be the $p$ th root of the sum of $p$ th powers. In particular, $l^{q}\left[l^{p}\right]$ consists (at least when $\left.p, q<\infty\right)$ of all sequences $a=\left(a_{n}\right)$ such that

$$
\|a\|_{l^{q}\left[{ }^{[p]}\right]}=\left\{\left|a_{0}\right|^{q}+\sum_{k=1}^{\infty}\left(\sum_{n=2^{k-1}}^{2^{k}-1}\left|a_{n}\right|^{p}\right)^{q / p}\right\}^{1 / q}<\infty .
$$

We shall usually use the more common notations $l^{p, q}$ and $\|\cdot\|_{p, q}$ in place of $l^{q}\left[l^{p}\right]$ and $\|\cdot\|_{l q\left[l^{p}\right]}$. We similarly define $l^{p, q, r}=l^{r}\left[l^{q}\left[l^{p}\right]\right]$. Observe that $l^{p, p}=l^{p}$.

The following elementary lemma (which will be used without comment later) gives an alternative description of $l^{r, \infty}$; we leave the proof as an exercise to the reader.

LEMMA 1. For a non-negative sequence $\left(a_{k}\right)$, the following statements are equivalent:

(a) There exists a constant $A \geq 0$ such that $\sum_{k=2^{n}}^{2^{n+1}}-1 \quad a_{k} \leq A$. 
(b) $\sum_{k=1}^{N} k^{t} a_{k}=O\left(N^{t}\right)$ (for any fixed $t>0$ ).

Thus, for $s>0, t \in \mathbf{R},\left(n^{t} \lambda_{n}\right) \in l^{r, \infty}$ if and only if $\sum_{n=1}^{N} n^{t r+s}\left|\lambda_{n}\right|^{r}=O\left(N^{s}\right)$.

We finish this section with a useful characterization of the lacunary series which lie in $H^{p}$ and $A^{p}$. Part (a) is well-known (see, for instance, Theorem I.V.6.4 in [45]), while (b) was proved in [8].

LEMMA A. Suppose $f(z)=\sum_{k=0}^{\infty} a_{2^{k}} z^{2^{k}}$. Then

(a) If $0<p \leq 2$, then $f \in H^{p}$ if and only if $\sum_{k=0}^{\infty}\left|a_{2^{k}}\right|^{2}<\infty$.

(b) If $0<p<\infty$, then $f \in A^{p}$ if and only if $\sum_{k=1}^{\infty} n_{k}^{-1}\left|a_{2^{k}}\right|^{p}<\infty$.

\section{Multipliers BetWeEn SPACES OF Bergman TYPE}

The characterization of $\left(A_{\alpha}^{p}, A_{\beta}^{q}\right)$ is trivial when $p=q=2$, but otherwise much more difficult. In some cases, such as $p=1, q=2$, or $q \leq 2 \leq p$, it is possible to give an explicit characterization. In a number of other cases, it seems that one can only hope to get conditions that are either sufficient or necessary, but not both.

The case $p=q=2$ is trivial because

$$
\|f\|_{A_{\alpha}^{2}}^{2}=\sum_{n=0}^{\infty} B(n+1, \alpha+1)\left|a_{n}\right|^{2} .
$$

By Stirling's formula, this sum is comparable to $\sum_{n=1}^{\infty}\left|a_{n}\right|^{2} n^{-1-\alpha}$. It follows that if $\alpha, \beta>$ -1 , then $\left(\lambda_{n}\right) \in\left(A_{\alpha}^{2}, A_{\beta}^{2}\right) \Leftrightarrow\left(\lambda_{n} n^{(\alpha-\beta) / 2}\right) \in l^{\infty}$, i.e. $\lambda \in D^{(\beta-\alpha) / 2} l^{\infty}$.

In [8], the characterization of multiplier spaces of the form $\left(A_{\alpha}^{p}, Y\right)$ or $\left(X, A_{\beta}^{q}\right)$ is reduced to the case $\alpha=\beta=0$, so we shall confine ourselves to looking at unweighted Bergman spaces for the rest of this section.

Wojtaszczyk [42] characterized $\left(A^{p}, A^{q}\right)$ for $q \leq 2 \leq p$; simpler proofs in more general situations were subsequently provided by Anderson [3], Blasco [7], and Jevtić-Jovanović [24]. This last paper also proves an analogous result for $H^{p}$. To summarize, we have the following.

THEOREM B. If $0<q \leq 2 \leq p<\infty$ and $1 / r=1 / q-1 / p$, then $\left(A^{p}, A^{q}\right)=D^{1 / r} l^{\infty, r}$ and $\left(H^{p}, H^{q}\right)=l^{\infty}$.

Corollary 9 of $[42]$ says that if $p \leq 2$, then $\left(\mathcal{B}, A^{p}\right)=D^{1 / p} l^{\infty, p}$. The above theorem allows us to slightly strengthen this.

COROLLARY 2. For $1<p \leq 2,\left(\mathcal{B}_{0}, A^{p}\right)=\left(\mathcal{B}, A^{p}\right)=D^{1 / p} l^{\infty, p}$. 
Proof. By Theorem B, $\left(A^{p^{\prime}}, A^{1}\right)=D^{1 / p} l^{\infty, p}$, so it suffices to prove that $\left(\mathcal{B}_{0}, A^{p}\right) \subseteq\left(A^{p^{\prime}}, A^{1}\right)$. Fix $\lambda=\left(\lambda_{n}\right) \in\left(\mathcal{B}_{0}, A^{p}\right)$, and let $T_{\lambda}^{*}: A^{p^{\prime}} \rightarrow A^{1}$ be the dual of $T_{\lambda}$. For any $a=\left(a_{n}\right) \in \mathcal{B}_{0}$, $b^{\prime}=\left(b_{n}^{\prime}\right) \in\left(A^{p}\right)^{\prime}=A^{p^{\prime}}$, let $T_{\lambda}^{*}\left(b^{\prime}\right)=\left(c_{n}^{\prime}\right) \in A^{1}$. Then

$$
\sum \lambda_{n} a_{n} \overline{b_{n}^{\prime}}(n+1)^{-1}=<T_{\lambda}(a), b^{\prime}>=<a, T_{\lambda}^{*}\left(b^{\prime}\right)>=\sum a_{n} \overline{c_{n}^{\prime}}(n+1)^{-1}
$$

which implies that $T_{\lambda}^{*}\left(b^{\prime}\right)=\left(\overline{\lambda_{n}} b_{n}^{\prime}\right)$, and so $T_{\lambda}^{*}=T_{\bar{\lambda}}$ is also a multiplier operator. By Theorem $\mathrm{B},\left(A^{p^{\prime}}, A^{1}\right)$ is closed under complex conjugation and so we are done.

Since $A^{p}$ is closed under the conjugation operator $\left(a_{n}\right) \mapsto\left(\overline{a_{n}}\right)$ and, as previously mentioned, $\left(A^{p}\right)^{*}=A^{p^{\prime}}$, it follows that $\left(A^{p}, A^{q}\right)=\left(A^{q^{\prime}}, A^{p^{\prime}}\right)$, for all $1<p, q<\infty$. In a similar fashion (but by a different pairing) one obtains $\left(H^{p}, H^{q}\right)=\left(H^{q^{\prime}}, H^{p^{\prime}}\right)$. These results will be useful later.

Elements of $H^{2}$ and $A^{2}$ are, of course, completely understood in terms of their Taylor coefficients. The "next best" spaces are $H^{1}$ and $A^{1}$, the largest Banach spaces among the unweighted Hardy and Bergman spaces respectively. We now state a theorem concerning the multiplier classes involving only $A^{p}$ and $H^{p}$ spaces of exponent 1 or 2. Since $A^{2}=D^{1 / 2} H^{2}$, it follows readily that $\left(H^{2}, Y\right)=D^{-1 / 2}\left(A^{2}, Y\right)$ and that $\left(X, H^{2}\right)=D^{1 / 2}\left(X, A^{2}\right)$ for any sequence spaces $X, Y$. Thus we may ignore those cases involving $H^{2}$, and our sixteen $a$ priori cases reduce to the following nine. This theorem is mostly a combination of results due to many different authors (as indicated by the proof); only the results (h), (i), and our proof of $(\mathrm{g})$ are new.

\section{THEOREM 3.}

(a) $\left(A^{2}, A^{2}\right)=l^{\infty}$.

(b) $\left(H^{1}, A^{2}\right)=D^{1 / 2} l^{2, \infty}$.

(c) $\left(A^{1}, A^{2}\right)=D^{-1 / 2} l^{2, \infty}$.

(d) $\left(A^{2}, A^{1}\right)=D^{1 / 2} l^{\infty, 2}$.

(e) $\left(A^{2}, H^{1}\right)=D^{-1 / 2} l^{\infty}$.

(f) $\left(A^{1}, A^{1}\right)=D^{-1} H_{1}^{1}$.

(g) $\left(A^{1}, H^{1}\right)=D^{-2} H_{1}^{1}$.

(h) $H(1,2,1) \subseteq\left(H^{1}, A^{1}\right) \subset H_{1}^{1}$.

(i) $D^{-1} H(1,2,1) \subset\left(H^{1}, H^{1}\right) \subseteq D^{-1} H_{1}^{1}$.

Proof. Part (a) is trivial. Part (b) is essentially equivalent to the fact that $\left(H^{1}, H^{2}\right)=l^{2, \infty}$, which is due to Hardy and Littlewood; see [12] and [21] for a proof, and also [31] for a more general discussion. Part (c) is due to Vukotic [40]. Parts (d) and (e) follow readily from Theorem B. Part (f) is due to Pavlović [33] (see [29] for a more general statement: $\left(A^{1}, A^{q}\right)=D^{-1} H_{1 / q}^{q}$ for all $\left.1 \leq q\right)$. 
Part (g) was proved in [29], but we shall demonstrate it using results of Blasco and Flett. This proof will also be useful for the last two parts of the theorem. Note first that $H^{1} \subseteq D^{-1} H(1,2,1)$, as proved in [19]. Therefore

$$
\left(A^{1}, H^{1}\right) \subseteq\left(A^{1}, D^{-1} H(1,2,1)\right)=D^{-1}(H(1,1,1), H(1,2,1))=D^{-2} H_{1}^{1},
$$

where the last equality is a special case of Theorem 5.3 of [7]. The opposite containment is quite similar, except that we begin with the inclusion $H^{1} \supset D^{-1} A^{1}=D^{-1} H(1,1,1)$, which was also proved in [19], and we end with $(H(1,1,1), H(1,1,1))=D^{-1} H_{1}^{1}$ which also follows from Theorem 5.3 of [7].

For $(\mathrm{h})$, we note first that

$$
\left(H^{1}, A^{1}\right) \supseteq\left(D^{-1} H(1,2,1), H(1,1,1)\right)=(H(1,2,1), H(1,1,2)) .
$$

The equality above follows from the trivial general fact that $(X, Y)=(D X, D Y)$ and the fact that $D^{s-t} H(p, q, t)=H(p, q, s)$, as long as $s, t>0$. This result, essentially due to Flett, is taken from [8]. Next, we note that Young's inequality for convolutions readily gives $H(1,2,1) \subseteq(H(1,2,1), H(1,1,2))$; for a detailed proof see Lemma $\mathrm{C}$ of [7]. We therefore deduce that $H(1,2,1) \subseteq\left(H^{1}, A^{1}\right)$.

As for the other containment, we first note that, as in $(\mathrm{g})$,

$$
\left(H^{1}, A^{1}\right) \subseteq\left(D^{-1} H(1,1,1), H(1,1,1)\right)=D^{1-1} H_{1}^{1}=H_{1}^{1} .
$$

To see that we do not have equality, note that the sequence $D^{1}$, corresponding to the Koebe function $z \mapsto z /(1-z)^{2}$, is obviously in $H_{1}^{1}$. However it does not lie in $\left(H^{1}, A^{1}\right)$, because $\sum_{n=1}^{\infty} n^{-1} z^{2^{n}} \in H^{1} \backslash D^{-1} A^{1}$ (see Lemma A).

Finally, we prove (i). First note that if we convolve (i.e., multiply coefficients of) a sequence in $\left(H^{1}, A^{1}\right)$ with one in $\left(A^{1}, H^{1}\right)$, we get a sequence in $\left(H^{1}, H^{1}\right)$. Since clearly $D^{-1} \in D^{-2} H_{1}^{1}=\left(A^{1}, H^{1}\right)$, we deduce from (h) that $D^{-1} H(1,2,1) \subseteq\left(H^{1}, H^{1}\right)$. We cannot have equality because the sequence whose coefficients are all 1 lies in $\left(H^{1}, H^{1}\right)$, but not in $D^{-1} H(1,2,1)$ (because the Koebe function does not lie in $H(1,2,1)$ ). To prove that $\left(H^{1}, H^{1}\right) \subseteq D^{-1} H_{1}^{1}$, we argue as in (g), (h) to first get the containment $\left(H^{1}, H^{1}\right) \subseteq$ $\left(D^{-1} H(1,1,1), D^{-1} H(1,2,1)\right)$, and finish as in $(\mathrm{g})$.

The following result, which follows from Theorem 4.2 of Blasco [7], generalizes part (c) of the above theorem; we shall use it later. We also state a corollary which follows by duality, as in Corollary 2.

LEMMA C. If $0<p \leq 1$, then $\left(A^{p}, A^{2}\right)=D^{3 / 2-2 / p} l^{2, \infty}$.

COROLLARY 4. $\left(A^{2}, \mathcal{B}_{0}\right) \subseteq\left(A^{1}, A^{2}\right)=\left(A^{2}, \mathcal{B}\right)$. Thus, $\left(A^{2}, \mathcal{B}_{0}\right) \subseteq D^{-1 / 2} l^{2, \infty}$.

Theorem 3 shows that one cannot in general hope for $\left(A^{p}, A^{q}\right)$ to have a simple description in terms of coefficients, since $H_{\alpha}^{p}$ is not solid if $p \neq 2$ (see Anderson-Shields [4] for more about solid spaces). In fact, $\left(A^{p}, A^{q}\right)$ is not solid if either $p, q<2$ or $p, q>2$; 
see [8]. If we wish for conditions involving $l^{p}$ or $l^{p, q}$, the best we can hope for are separate necessary and sufficient conditions in such cases.

We already understand $\left(A^{1}, A^{2}\right)$ and $\left(A^{2}, A^{2}\right)$ in terms of the mixed-norm sequences. For $1<p<2,\left(A^{p}, A^{2}\right)$ is, of course, solid but seems to be difficult to determine. A result of Blasco [7] says that it contains $D^{-1 / r} l^{r, \infty}$, where $1 / r=1 / p-1 / 2$. We now sharpen this result. Note that $l^{r, \infty, \infty}$ contains sequences that are not in $l^{s, t}$ unless $s=t=\infty$ (e.g. $\sum_{k=1}^{\infty} \sum_{j=0}^{k-1} x^{2^{k}+2^{j}}$ ).

THEOREM 5. Let $1<p<2$ and $\frac{1}{r}=\frac{1}{p}-\frac{1}{2}=\frac{2-p}{2 p}$. Then $D^{-1 / r} l^{r, \infty, \infty} \subset\left(A^{p}, A^{2}\right) \subset$ $D^{-1 / r} l^{\infty}$.

To prove this theorem, we need the following result from [37] and [30].

LEMMA D. Let $1<p<\infty$ and let $\Delta_{k}$ be the multiplier operator whose coefficients are 1 in the kth dyadic block $I_{k}$ and zero elsewhere. Then

$$
\|f\|_{A^{p}}^{p} \approx \sum_{k=0}^{\infty} 2^{-k}\left\|\Delta_{k} f\right\|_{H^{p}}^{p}
$$

We now give a couple of equivalent formulations of (2) that we shall use later; in particular the second will be the key to proving Theorem 5. First, it is easy to deduce that, for $1<p<\infty$,

$$
\|f\|_{A^{p}}^{p} \approx \sum_{k=0}^{\infty}\left\|\Delta_{k} f\right\|_{A^{p}}^{p}
$$

We state our second reformulation, which clarifies the relationship between $A^{p}$ and $H^{p}$ $(p>1)$, as a separate statement, which may have some independent interest. For more on the role of mixed-norm spaces in the study of multipliers, we refer the reader to the forthcoming works [9] and [10].

PROPOSITION 6. If $1<p<\infty$, then $\|f\|_{A^{p}}^{p} \approx|f(0)|^{p}+\sum_{k=0}^{\infty}\left\|\Delta_{k} D^{-1 / p} f\right\|_{H^{p}}^{p}$. Equivalently, $A^{p}=D^{1 / p} l^{p}\left[H^{p}\right]$.

Proof. Proposition 3.7 of [8] says that $B V \subset\left(H^{p}, H^{p}\right)$, and thus also $H^{p} \subset\left(B V, H^{p}\right)$, for $1<p<\infty$. Applying the Closed Graph Theorem to $B V$ multipliers from $H^{p}$ to itself, and to $H^{p}$ multipliers from $B V$ to $H^{p}$, we see that all of these multipliers are bounded. Therefore by the Uniform Boundedness Principle (applied to the family $\left\{T_{\lambda}:\|\lambda\|_{B V} \leq 1\right\}$ of multipliers from $H^{p}$ to itself), it follows that $\left\|\left(a_{n} b_{n}\right)\right\|_{H^{p}} \leq C\left\|\left(a_{n}\right)\right\|_{B V} \cdot\left\|\left(b_{n}\right)\right\|_{H^{p}}$.

To prove the lemma, we must show that the ratio of the quantities $2^{-k}\left\|\Delta_{k} f\right\|_{H^{p}}$ and $\left\|\Delta_{k} D^{-1 / p} f\right\|_{H^{p}}$ is bounded above and below, independently of $k$. To change one of these expressions to the other, we simply applied the multiplier operator $T_{k}$ given by the sequence $\Delta_{k}\left(2^{k / p} n^{-1 / p}\right)$, or its "inverse" $T_{k}^{-1}$ given by $\Delta_{k}\left(2^{-k / p} n^{1 / p}\right)$. Both of these sequences are in $B V$, the space of bounded variation sequences, with total variation at most $2^{1+1 / p}$, and so we are done.

Proof of Theorem 5. The fact that $\left(A^{p}, A^{2}\right) \subseteq D^{-1 / r} l^{\infty}$ is part of Theorem 4.7 of [8], so it suffices to prove the other containment. We claim that $D^{1 / 2-1 / p} l^{\infty}\left[\left(H^{p}, H^{2}\right)\right] \subset\left(A^{p}, A^{2}\right)$; 
actually, these two spaces are equal, but we omit the proof of the reverse containment (which is not difficult with the help of the Closed Graph Theorem), as it is of no relevance here.

By Proposition 6, we have $A^{p}=D^{1 / p} l^{p}\left[H^{p}\right]$. Discarding the fractional derivatives, our claim thus reduces to the inclusion $l^{\infty}\left[\left(H^{p}, H^{2}\right)\right] \subset\left(l^{p}\left[H^{p}\right], l^{2}\left[H^{2}\right]\right)$. Let $f \in l^{p}\left[H^{p}\right]$ and $\lambda \in l^{\infty}\left[\left(H^{p}, H^{2}\right)\right]$. Now, $\|f\|_{l^{p}\left[H^{p}\right]}=\left\|\left(\left\|S_{k} f\right\|_{H^{p}}\right)_{k=0}^{\infty}\right\| l_{l^{p}}$, and

$$
\begin{aligned}
\left\|T_{\lambda} f\right\|_{l^{2}\left[H^{2}\right]} & =\left\|\left(\left\|T_{S_{k} \lambda} S_{k} f\right\|_{H^{2}}\right)_{k=0}^{\infty}\right\|_{l^{2}} \\
& \leq\left\|\left(\left\|S_{k} \lambda\right\|_{\left(H^{p}, H^{2}\right)} \cdot\left\|S_{k} f\right\|_{H^{p}}\right)_{k=0}^{\infty}\right\|_{l^{2}} \\
& \leq\|\lambda\|_{\left.l \infty\left[l^{\infty} H^{p}, H^{2}\right)\right]} \cdot\left\|\left(\left\|S_{k} f\right\|_{H^{p}}\right)_{k=0}^{\infty}\right\|_{l^{2}} \\
& \leq\|\lambda\|_{l^{\infty}\left[\left(H^{p}, H^{2}\right)\right]} \cdot\left\|\left(\left\|S_{k} f\right\|_{H^{p}}\right)_{k=0}^{\infty}\right\| l_{l^{p}} \\
& =\|\lambda\|_{l^{\infty}\left[\left(H^{p}, H^{2}\right)\right]} \cdot\|f\|_{l^{p}\left[H^{p}\right]},
\end{aligned}
$$

proving our claim.

To finish the proof, we appeal to a theorem of Hedlund [22] which says that $l^{r, \infty}$ is a proper subset of $\left(H^{p}, H^{2}\right)$, for all $1<p<2$. Consequently, $\left(A^{p}, A^{2}\right)$ properly contains $D^{1 / 2-1 / p} l^{\infty}\left[l^{r, \infty}\right]=D^{-1 / r} l^{r, \infty, \infty}$, as required.

Various theorems on the Taylor coefficients of $H^{1}$ functions are known. To state one that is due to Paley, let us first make a couple of definitions. A sequence of positive integers $\left(n_{k}\right)$ is lacunary if there exists $Q>1$ such that $n_{k+1} / n_{k} \geq Q, k \in \mathrm{N}$, and it is sparse if it has at most $N$ terms in each dyadic block, for some fixed $N>1$. Clearly, every lacunary sequence is sparse. The converse is false (e.g. $\left.\sum_{n=1}^{\infty}\left(x^{2^{n}}+x^{2^{n}+1}\right)\right)$; in fact, sparse sequences are precisely finite sums of lacunary sequences. We shall say that a subset $E$ of $N$ (viewed as a subsequence) has the $H$-Paley property if for every $\left(a_{n}\right) \in H^{1}$, we have $\sum_{n \in E}\left|a_{n}\right|^{2}<\infty$.

Paley's theorem (cf. [12], p. 104, for example) says that every lacunary sequence $E \subset \mathrm{N}$ has the H-Paley property. The theorem remains valid if $E$ is merely sparse, since we can partition the terms of a sparse sequence into a finite collection of lacunary subsequences. W. Rudin [35] proved the converse, and so we have the following result.

THEOREM E. A sequence $E \subset \mathrm{N}$ has the H-Paley property if and only if it is sparse.

A subset $E$ of $\mathrm{N}$ has the H-Paley property if and only if $\lambda_{E} \in\left(H^{1}, H^{2}\right)$, where $\lambda_{E}$ is given by the formal power series $\sum_{n \in E} x^{n}$. Since $\left\|\sum_{n \in E} x^{n}\right\|_{i^{2, \infty}}=\sup _{k}\left|I_{k} \cap E\right|^{1 / 2}$, the Paley-Rudin Theorem is thus seen to be a special case of Theorem 3(b) (or more precisely of its equivalent form $\left.\left(H^{1}, H^{2}\right)=l^{2, \infty}\right)$.

A similar argument gives an $A^{1}$ analogue of the Paley-Rudin theorem. To be precise, let us say that a subset $E$ of $\mathrm{N}$ has the $A$-Paley property if for every $\left(a_{n}\right) \in A^{1}$, we have $\sum_{n \in E} n^{-1}\left|a_{n}\right|^{2}<\infty$. As a corollary of Theorem 3(c), we obtain the following new result.

THEOREM 7. A sequence $E \subset \mathrm{N}$ has the A-Paley property if and only if it is sparse.

We remark that for analytic functions whose Taylor coefficients form a sparse sequence, or a sequence monotonic on dyadic blocks, there is a characterization for their membership in any of the spaces $A^{p}$; see Propositions 2.1 and 2.4 of Buckley-Koskela-Vukotić [8]. 


\section{ESSENTIAL NORMS AND COMPACTNESS OF MULTIPLIERS}

Compact multipliers on Bergman spaces have not received much attention. This section attempts to provide some information on this.

Recall that the essential norm $\|T\|_{e}$ of a bounded operator $T$ between the Banach spaces $X$ and $Y$ is given by

$$
\|T\|_{e}=\inf \{\|T-K\|: K \in \mathcal{K}(X, Y)\}
$$

and provides a measure of the noncompactness of $T$. Clearly, $T$ is compact if and only if $\|T\|_{e}=0$. The essential norm can actually be computed explicitly in certain cases. We begin with a simple example where this is so.

PROPOSITION 8. The essential norm of $T_{\lambda} \in \mathcal{L}\left(A_{\alpha}^{2}, A_{\beta}^{2}\right)$ is given by

$$
\left\|T_{\lambda}\right\|_{e}=\limsup _{n \rightarrow \infty}\left|\lambda_{n}\right|\left(\frac{B(n+1, \beta+1)}{B(n+1, \alpha+1)}\right)^{1 / 2} \text {. }
$$

In particular, $T_{\lambda}$ is a compact operator if and only if $\left(\left|\lambda_{n}\right| n^{(\alpha-\beta) / 2}\right) \in c_{0}$.

Proof. Let $U_{\alpha}$ be the multiplier operator given by the sequence $\left(B(n+1, \alpha+1)^{1 / 2}\right)$. It follows from $(1)$ that $U_{\alpha}$ is an isometry from $A_{\alpha}^{2}$ to $l^{2}$. Consequently, $\|T\|_{A_{\alpha}^{2} \rightarrow A_{\beta}^{2}}=\left\|U_{\beta} T U_{\alpha}^{-1}\right\|_{l^{2} \rightarrow l^{2}}$ for any operator $T$, and so it suffices to show that $\left\|T_{\lambda}\right\|_{e}=\lim \sup \left|\lambda_{n}\right|$, for any multiplier operator $T_{\lambda} \in \mathcal{L}\left(l^{2}, l^{2}\right)$.

Suppose therefore that $T_{\lambda} \in \mathcal{L}\left(l^{2}, l^{2}\right)$. For an arbitrary positive integer $N$, we denote by $T_{N}$ the multiplier operator corresponding to the sequence whose first $N$ terms are $\lambda_{1}, \lambda_{2}$, $\ldots, \lambda_{N}$, and whose remaining terms are $0 . T_{N}$ is compact since it has finite rank. Therefore

$$
\left\|T_{\lambda}\right\|_{e}^{2} \leq\left\|T_{\lambda}-T_{N}\right\|^{2}=\sup \left\{\left(\sum_{n>N}\left|\lambda_{n}\right|^{2}\left|a_{n}\right|^{2}:\left\|\left(a_{n}\right)\right\|_{l^{2}}^{2} \leq 1\right\} \leq \sup _{n>N}\left|\lambda_{n}\right|^{2} .\right.
$$

Since $N$ is arbitrary, we are done.

For the lower bound on $\left\|T_{\lambda}\right\|_{e}$, let us suppose that $K \in \mathcal{K}\left(l^{2}, l^{2}\right)$ and that $\left(\lambda_{n_{k}}\right)$ is a subsequence of $\left(\lambda_{n}\right)$ such that $\lim _{k \rightarrow \infty}\left|\lambda_{n_{k}}\right|=L=\lim \sup \left|\lambda_{n}\right|$. Let $\mu_{k} \in l^{2}$ be the sequence whose $n_{k}$ th term is 1 and all other terms are zero. Then $\left\|T_{\lambda} \mu_{k}\right\|_{l^{2}} \rightarrow L$ as $k \rightarrow \infty$, but $K \mu_{k} \rightarrow 0$ in $l^{2}$, since $\left(\mu_{k}\right)$ tends to zero weakly in $l^{2}$. Thus $\left\|T_{\lambda}-K\right\|_{l^{2} \rightarrow l^{2}} \geq L$. Since $K$ is arbitrary, we conclude that $\left\|T_{\lambda}\right\|_{e} \geq L$, as required.

REMARK. Recall (see Pietsch [34]) that, for two Banach spaces $E$ and $F$ and a bounded linear operator $T \in L(E, F)$, the non-increasing sequence $\left(a_{n}(T)\right)$ of approximation numbers is defined by

$$
a_{n}(T)=\inf \left\{\left\|T-A_{n}\right\|: A_{n} \in L(E, F), \operatorname{rank} A_{n} \leq n\right\} .
$$

In general, $\|T\|_{e} \leq \lim a_{n}(T)$, and if $E, F$ are both Hilbert spaces then $\|T\|_{e}=\lim a_{n}(T)$. Also, if $E, F$ are Hilbert spaces then $T \in L(E, F)$ belongs to the Schatten class $S_{p}$ exactly 
when $\left(a_{n}(T)\right) \in l_{p}$ and if $E, F$ are Banach spaces, then $T \in L(E, F)$ is strongly nuclear (see [34]) exactly when $\left(a_{n}(T)\right)$ is rapidly decreasing, i.e. for each $k, \lim n^{k} a_{n}(T)=0$.

Let $\mu_{n}=\left|\lambda_{n}\right|\left(\frac{B(n+1, \beta+1)}{B(n+1, \alpha+1)}\right)^{1 / 2}$ and let $\nu_{n}=\sup _{m>n} m^{(\alpha-\beta) / 2}\left|\lambda_{m}\right|$. By the above proof, we have $a_{N}\left(T_{\lambda}\right) \leq \sup _{n>N} \mu_{n}$, and thus $T_{\lambda} \in L\left(A_{\alpha}^{2}, A_{\beta}^{2}\right)$ is in $S_{p}$ (or is strongly nuclear) if $\nu \in l_{p}$ (resp., $\nu$ is rapidly decreasing). In particular, $T_{\lambda}$ is a trace class operator if $\nu \in l_{1}$, and is Hilbert-Schmidt if $\nu \in l_{2}$.

Proposition 3.7 of [8] says that any $\left(\lambda_{n}\right) \in B V$ gives a bounded self-multiplier of $A^{p}$, for any $1<p<\infty$. We now compute the essential norm of such an operator.

THEOREM 9. Let $1<p<\infty$ and let $T \in \mathcal{L}\left(A^{p}, A^{p}\right)$ be the operator associated with the sequence $\left(\lambda_{n}\right) \in B V$, where $\lim _{n \rightarrow \infty} \lambda_{n}=L$. Then $\|T\|_{e}=|L|$. Consequently, $T \in \mathcal{K}\left(A^{p}, A^{p}\right)$ if and only if $L=0$. If $T$ is bounded, but not compact, then the operator $T_{\lambda-L}$ is a closest compact approximant.

Proof. We begin by proving that $\|T\|_{e} \geq|L|$. The functions $f_{n}(z)=(n p / 2+1)^{1 / p} z^{n}$ lie on the unit sphere of $A^{p}$ and the sequence $\left(f_{n}\right)$ converges to zero uniformly on compact subsets of the disk. The Lebesgue Dominated Convergence Theorem and the representation of linear functionals on $A^{p}$ for $p>1$ imply that this sequence weakly converges to zero, and so $K f_{n} \rightarrow 0$ for any $K \in \mathcal{K}\left(A^{p}, A^{p}\right)$. Therefore we obtain

$$
\begin{aligned}
\|T-K\| & \geq \limsup _{n \rightarrow \infty}\left\|T f_{n}-K f_{n}\right\|_{p} \\
& \geq \limsup _{n \rightarrow \infty}\left(\left\|T f_{n}\right\|_{p}-\left\|K f_{n}\right\|_{p}\right) \\
& =\limsup _{n \rightarrow \infty}\left\|T f_{n}\right\|_{p} \\
& =\limsup _{n \rightarrow \infty}\left|\lambda_{n}\right|=|L|
\end{aligned}
$$

which implies $\|T\|_{e} \geq|L|$.

The reverse inequality is equivalent to showing that $T$ is compact if $L=0$, so we assume that $L=0$ below. We may also assume without loss of generality that $\|T\| \leq 1$, since the theorem is invariant under multiplication of $\lambda$ by a non-zero constant.

If $f \in A^{p}$ and $f=g+h$, where $g$ is a Taylor polynomial of $f$ whose order is a power of 2 , then Lemma $D$ implies that $\|f\|_{A^{p}} \approx\|g\|_{A^{p}}+\|h\|_{A^{p}}$ (in fact, the power-of-2 restriction is unnecessary). We denote by $F_{n}$ the linear functional that takes any $f \in A^{p}$ to its $n$th Taylor coefficient; $F_{n}$ is bounded by the Cauchy integral formula.

Suppose now that $\left(f_{m}\right)$ is a sequence of functions in the unit ball of $A^{p}$. Defining $\Delta_{k}$ as in Lemma $\mathrm{D}$, we see that the functions $T f_{m}$ and $\Delta_{k} T f_{m}$ are in the unit ball for all $m \geq 1, k \geq 0$. By finite dimensionality, each of the sequences $\left(\Delta_{k} T f_{m}\right)$ has a subsequence convergent in $A^{p}$. By a diagonalization argument, we can produce a subsequence of $\left(f_{m}\right)$ such that each of the associated sequences $\left(\Delta_{k} T f_{m}\right)$ is convergent. Let us also denote this diagonal subsequence by $\left(f_{m}\right)$ (since we have no more use for the discarded functions). By finite dimensionality (and the boundedness of every $F_{n}$ ), the convergence of $\left(\Delta_{k} T f_{m}\right)$ in $A^{p}$ and in $l^{\infty}$ are (topologically) equivalent. We therefore have a formal limit sequence $\left(b_{n}\right)$ such that $F_{n}\left(T f_{m}\right) \rightarrow b_{n}(m \rightarrow \infty)$ for all $n$. In fact, since each of the functions $\Delta_{k} T f_{m}$ is tending 
to $\Delta_{k}\left(b_{n}\right)$ (this last function being defined in the obvious manner, even though we do not yet know that $\left(b_{n}\right)$ represents a function), it follows from (3) by an easy limiting argument that $\left(b_{n}\right) \in A^{p}$. We denote the associated limiting function by $g$.

It remains to check that $\left(T f_{m}\right)$ converges to $g$ in $A^{p}$. Since $B V \subseteq\left(A^{p}, A^{p}\right)$, we deduce from the Closed Graph Theorem and the Uniform Boundedness Principle that $\left\|T_{\mu}\right\|_{A^{p} \rightarrow A^{p}} \leq C\|\mu\|_{B V}$ (a formally identical argument is given in the proof of Proposition 6).

Suppose $\epsilon>0$ is given, and let $k=k(\epsilon)$ be a fixed positive integer to be specified later. We write $\lambda=\lambda_{1}+\lambda_{2}$, where the $n$th term of the sequence $\lambda_{1}$ equals that of $\lambda$ if $n<2^{k}$, and equals 0 otherwise. Now if $k$ is sufficiently large, then $\left\|\lambda_{2}\right\|_{B V}$ is very small, so we can assume that $k$ was chosen so large that $\left\|T_{\lambda_{2}}\right\| \leq \epsilon$. Splitting $g=g_{1}+g_{2}$ in the same manner, we see there is some $C=C(p)$ such that $\left\|T_{\lambda_{2}} f_{m}-g_{2}\right\|_{A^{p}} \leq C \epsilon$ for all $m$. But by finite dimensionality, we obviously have $\left\|T_{\lambda_{1}} f_{m}-g_{1}\right\|_{A^{p}} \leq \epsilon$ for sufficiently large $m$. Thus $\left(T f_{m}\right)$ converges in $A^{p}$ to $g$, and $T$ is compact as required.

The other statements in the theorem are immediate. I

The results above illustrate that some of the known characterizations of (bounded) multipliers allow for an easy compactness analogue. A familiar "rule of thumb" suggests that changing a "big-O" condition for boundedness to a "little-o" condition gives rise to a compactness condition. In this section, we derive several compactness results from the results on the boundedness, guided by this principle.

The following result of Duren [13] will be needed later.

THEOREM F. If $0<p \leq 2 \leq q<\infty$ and $\lambda_{n}=O\left(n^{1 / q-1 / p}\right)$, then $T_{\lambda} \in \mathcal{L}\left(H^{p}, H^{q}\right)$ and the exponent is best possible.

The compactness analogue of the above result follows readily (but was not stated in [13]). From its proof, we learn that, using Proposition 8 , the rule of thumb is valid in particular if we can factorize a bounded operator through $H^{2}$ (or $A^{2}$ ).

COROLLARY 10. Let $0<p \leq 2 \leq q<\infty$. If $\lambda_{n}=o\left(n^{1 / q-1 / p}\right)$, then $T_{\lambda} \in \mathcal{K}\left(H^{p}, H^{q}\right)$.

Proof. Let $\lambda=\left(c_{n} n^{1 / q-1 / p}\right), c_{n}=o(1)$. By Theorem $F, D^{1 / 2-1 / p} \in \mathcal{L}\left(H^{p}, H^{2}\right)$ and $D^{1 / q-1 / 2} \in \mathcal{L}\left(H^{2}, H^{q}\right)$. One easily verifies that $T_{c} \in \mathcal{K}\left(H^{2}, H^{2}\right)$. Thus $T_{\lambda}$ is compact, since it is a composition of three bounded operators, one of which is compact.

We now recall a Bergman space analogue of Duren's theorem; part (a) is from [40], and (b) is from [7]. In each case, the exponent is best possible.

THEOREM G. Suppose $0<p \leq 2 \leq q<\infty$ and $1 / r=1 / p-1 / q$.

(a) If $\lambda_{n}=O\left(n^{-2 / r}\right)$, then $T_{\lambda} \in \mathcal{L}\left(A^{p}, A^{q}\right)$.

(b) If $p \geq 1$ and $\left(n^{1 / r} \lambda_{n}\right) \in l^{r, \infty}$ then $T_{\lambda} \in \mathcal{L}\left(A^{p}, A^{q}\right)$.

Here is a little-oh compactness version, apparently not considered before.

THEOREM 11. Suppose $0<p \leq 2 \leq q<\infty$ and $1 / r=1 / p-1 / q$.

(a) If $\lambda_{n}=o\left(n^{-2 / r}\right)$, then $T_{\lambda} \in \mathcal{K}\left(A^{p}, A^{q}\right)$.

(b) If $p \geq 1$ and $\sum_{2^{k} \leq n<2^{k+1}}\left|\lambda_{n}\right|^{r}=o\left(2^{-k}\right)$ then $T_{\lambda} \in \mathcal{K}\left(A^{p}, A^{q}\right)$. 
Proof. Part (a) follows immediately by $A^{2}$ factorization, so we prove only (b). We shall write $T_{\lambda}=T_{3} \circ T_{2} \circ T_{1}$, where $T_{1} \in \mathcal{L}\left(A^{p}, A^{q}\right), T_{2} \in \mathcal{K}\left(A^{2}, A^{2}\right)$, and $T_{3} \in \mathcal{L}\left(A^{2}, A^{q}\right)$. Let $I_{k}=\left\{n: 2^{k} \leq n<2^{k+1}\right\}$ and $\mu_{k}=2^{k} \sum_{n \in I_{k}}\left|\lambda_{n}\right| r$. Clearly, $\mu_{k}=o(1)$. Now let $s^{-1}=p^{-1}-2^{-1}, t^{-1}=2^{-1}-q^{-1}$, and so $r^{-1}=s^{-1}+t^{-1}$. Define $\alpha_{n}=\mu_{k}^{-1 / s}\left|\lambda_{n}\right|^{r / s}$ and $\beta_{n}=\mu_{k}^{-1 / t}\left|\lambda_{n}\right|^{r / t}$ whenever $n \in I_{k}$ (with $\alpha_{n}=0=\beta_{n}=0$ if $\mu_{k}=0$ ). By Theorem $\mathrm{G}(\mathrm{b})$, $\left(n^{1 / s} \alpha_{n}\right) \in l^{s, \infty}$ and $\left(n^{1 / t} \beta_{n}\right) \in l^{t, \infty}$, and so $\left(\alpha_{n}\right) \in\left(A^{p}, A^{2}\right),\left(\beta_{n}\right) \in\left(A^{2}, A^{q}\right)$. The sequence $\left(\operatorname{sgn}\left(\lambda_{n}\right) \cdot \mu_{k}^{1 / r}\right)$ lies in $c_{0}=\mathcal{K}\left(A^{2}, A^{2}\right)$. Since

$$
\lambda_{n}=\alpha_{n} \cdot\left[\operatorname{sgn}\left(\lambda_{n}\right) \cdot \mu_{k}^{1 / r}\right] \cdot \beta_{n},
$$

we are done.

Let us also note that the study of compact operators between weighted Bergman spaces reduces to the unweighted case. In fact if $p, q \geq 0, \alpha, \beta \in \mathbf{R}, \mu=\left(\mu_{n}\right)=\left(n^{\alpha / p-\beta / q} \lambda_{n}\right)$, then $T_{\lambda} \in \mathcal{K}\left(A_{\alpha}^{p}, A_{\beta}^{q}\right)$ if and only if $T_{\mu} \in \mathcal{K}\left(A^{p}, A^{q}\right)$. This compactness analogue of Corollary $1.4(\mathrm{~d})$ in [8] follows from part (a) of that corollary in the same manner, since the composition of a compact and a bounded operator is compact.

We shall now obtain the compactness versions of parts (b) and (c) of Theorem 3. To this end, we use the following elementary lemma based on J.E. McCarthy's suggestion.

LEMMA 12. Let $\left(c_{n}\right)$ be a nonnegative sequence such that $\sum_{n=1}^{N} c_{n}=o(N)$. Then there exists a positive sequence $\left(\mu_{n}\right)$ such that $\mu_{n} \rightarrow \infty$ and $\sum_{n=1}^{N}\left|\mu_{n}\right|^{2} c_{n}=O(N)$.

Proof. Let $\gamma_{m}=\sum_{k=1}^{m} c_{k} / m$, and define $\delta_{n}=\sup \left\{\gamma_{m}: m \geq n\right\}$. Then $\mu_{n}=\delta_{n}^{-1 / 2}$ has the desired properties: $\mu_{n}>0, \mu_{n} \rightarrow \infty$ (since $\gamma_{n} \rightarrow 0$ ), and

$$
\sum_{n=1}^{N}\left|\mu_{n}\right|^{2} c_{n}=\sum_{n=1}^{N} \frac{c_{n}}{\delta_{n}} \leq \frac{1}{\gamma_{N}} \sum_{n=1}^{N} c_{n}=N .
$$

\section{THEOREM 13.}

(a) $T_{\lambda} \in \mathcal{K}\left(H^{1}, H^{2}\right) \Leftrightarrow \sum_{n=1}^{N} n^{2}\left|\lambda_{n}\right|^{2}=o\left(N^{2}\right)$.

(b) $T_{\lambda} \in \mathcal{K}\left(A^{1}, A^{2}\right) \Leftrightarrow \sum_{n=1}^{N} n^{2}\left|\lambda_{n}\right|^{2}=o(N)$.

Proof. We prove only (b); part (a) is similar.

$(\Leftarrow)$ : Suppose that $\sum_{n=1}^{N} n^{2}\left|\lambda_{n}\right|^{2}=o(N)$. Apply Lemma 12 with $c_{n}=\left|\lambda_{n}\right|^{2} n^{2}$ and obtain the corresponding sequence $\left(\mu_{n}\right)$. By Theorem 3 (c), we have $T_{\lambda \mu} \in \mathcal{L}\left(A^{1}, A^{2}\right)$, while Proposition 8 implies that $T_{1 / \mu} \in \mathcal{K}\left(A^{2}, A^{2}\right)$. Thus, $T_{\lambda}=T_{\lambda \mu} \circ T_{1 / \mu} \in \mathcal{K}\left(A^{1}, A^{2}\right)$.

$(\Rightarrow)$ : Let $T_{\lambda} \in \mathcal{K}\left(A^{1}, A^{2}\right)$. For $0<R<1$ consider $f_{R}(z)=(1-R)^{3 / 2}(1-R z)^{-7 / 2}$. One sees that

$$
\begin{aligned}
\left\|f_{R}\right\|_{A^{1}} & \sim(1-R)^{3 / 2} \int_{0}^{1} \int_{0}^{2 \pi} \frac{d \theta}{\left|1-R r e^{i \theta}\right|^{7 / 2}} r d r \\
& \leq C(1-R)^{1 / 2} \int_{0}^{1}(1-R r)^{-3 / 2} d r \leq C .
\end{aligned}
$$


Hence, $\left\{T_{\lambda} f_{R}\right\}$ is a relatively compact family in $A^{2}$, and any subfamily of it contains a subsequence $\left(T_{\lambda} f_{R_{n}}\right)$ which converges in the $A^{2}$-norm. Also,

$$
T_{\lambda} f_{R}(z)=(1-R)^{3 / 2} \sum_{n} \lambda_{n} a_{n} R^{n} z^{n}, \quad a_{n} \sim A n^{5 / 2} .
$$

One easily sees that $T_{\lambda} f_{R} \rightarrow 0$ as $R \rightarrow 1$, uniformly on compact subsets. To get this, we use Theorem $3(\mathrm{c})$ to get the crude estimate $\lambda_{n}=O\left(n^{-1 / 2}\right)$. Together with the above estimate on $a_{n}$, we see that $\lambda_{n} a_{n}=O\left(n^{2}\right)$. On each fixed compact disk $\{z:|z| \leq \rho\} \subset \mathrm{D}$, $0<\rho<1$, this yields

$$
\left|T_{\lambda} f_{R}\right| \leq C(1-R)^{3 / 2} n^{2} \rho^{n} \rightarrow 0, \quad R \rightarrow 1^{-} .
$$

A subsequence $\left(T_{\lambda} f_{R_{n}}\right)$ converges in the $A^{2}$-norm, while 0 is the only cluster point for the normal family $\left\{T_{\lambda} f_{R}\right\}$ : this yields $T_{\lambda} f_{R} \rightarrow 0$ in the norm of $A^{2}$ on all subsequences. We also have, for any $0<R<1$,

$$
\left\|T_{\lambda} f_{R}\right\|_{A^{2}}^{2} \sim C(1-R)^{3} \sum_{n}\left|\lambda_{n}\right|^{2} n^{4} R^{2 n}
$$

Taking $R=1-1 / N$, and truncating the sum at the $N$ th place, we obtain $N^{-3} \sum_{n=1}^{N}\left|\lambda_{n}\right|^{2} n^{4}$ $\rightarrow 0$, and so $\sum_{n=1}^{N}\left|\lambda_{n}\right|^{2} n^{2}=o(N)$.

We now discuss when the bounded and compact operators between $A^{p}$ spaces coincide, following a suggestion of $\mathrm{P}$. Wojtaszczyk. This theorem implies, in particular, that the $\left(A^{p}, A^{q}\right)$ multipliers $(p \geq 2 \geq q)$ described in [42] are all compact.

THEOREM 14. Suppose $p>1, q \geq 1$. Then $\mathcal{L}\left(A^{p}, A^{q}\right)=\mathcal{K}\left(A^{p}, A^{q}\right)$ if and only if $q<p$. In fact, there exists a non-compact multiplier operator for all $p \leq q$.

To prove this result, we shall need a couple of preliminary lemmas. The first is due to Lindenstrauss-Pełczyński [27] for $p>1$, and to Shields-Williams [36] for $p=1$ (see also [41], Section III.A). The second is a special case of Proposition 2.4 in [8].

LEMMA H. If $p \geq 1$, then $A^{p}$ is topologically isomorphic to $l^{p}$, i.e. there exists a linear one-to-one correspondence between these spaces which is continuous in both directions.

LEMMA I. Suppose that $a_{n} \geq 0,1<p, C<\infty$, that $\left(a_{n}\right)_{n \in I_{k}}$ is monotonic for all $k$, and that $a_{m} \leq C a_{n}$, for all $n, m \in I_{k}, k>0$. Then $\left(a_{n}\right) \in A^{p}$ if and only if $\sum_{n=1}^{\infty} n^{p-3}\left|a_{n}\right|^{p}<\infty$. In fact, $\left\|\left(a_{n}\right)\right\|_{A^{p}}^{p} \approx \sum_{n=1}^{\infty} n^{p-3}\left|a_{n}\right|^{p}$.

Proof of Theorem 14. A theorem of Pitt (cf. [26], pp. 208-209) states that $\mathcal{L}\left(l^{p}, l^{q}\right)=$ $\mathcal{K}\left(l^{p}, l^{q}\right)$ for all $1 \leq q<p$. Thus if $q<p$, the compactness of $T \in \mathcal{L}\left(A^{p}, A^{q}\right)$ follows by using Lemma $\mathrm{H}$ to factorize it through $l^{p}$ and $l^{q}$.

It remains to construct a non-compact $\left(A^{p}, A^{q}\right)$ multiplier whenever $1<p \leq q$. The case $p=q$ is easily handled, since the identity map $T_{1}$ cannot be compact on an infinite dimensional space, but obviously $T_{1} \in \mathcal{L}\left(A^{p}, A^{p}\right)$. We may therefore assume that $p<q$. 
Let $\lambda=\left(n^{2 / q-2 / p}\right)$. It follows from Theorem 1.3(a) of [8] that $T_{\lambda} \in \mathcal{L}\left(A^{p}, A^{q}\right)$, but we shall see that $T_{\lambda} \notin \mathcal{K}\left(A^{p}, A^{q}\right)$. Let $\Delta_{k}$ be as in Lemma $\mathrm{D}$ and define $f_{k}(z)=\sum_{n \in I_{k}} n^{2 / p-1} z^{n}$. By Lemma I, $\left\{f_{k}\right\}$ is a bounded subset of $A^{p}$. Suppose that a subsequence of $\left(T_{\lambda} f_{k}\right)$ is norm-convergent to some $g \in A^{q}$. Recalling that $F_{n}$, the linear functional that takes $f$ to its $n$th Taylor coefficient, is bounded on $A^{q}$, it follows readily that all the Taylor coefficients of $g$ must be zero, and so $g \equiv 0$. But this is impossible, since $\left(T_{\lambda} f_{k}\right)(z)=\sum_{n \in I_{k}} n^{2 / q-1} z^{n}$ is bounded away from zero in $A^{q}$ by Lemma 1 . I

COROLLARY 15. Let $1 \leq p<2$ and $\frac{1}{r}=\frac{1}{p}-\frac{1}{2}=\frac{2-p}{2 p}$. Then

(a) $D^{1 / 2} l^{\infty, r} \subset \mathcal{K}\left(H^{p}, A^{p}\right)$

(b) $D^{-1 / r} l^{\infty, r} \subset \mathcal{K}\left(A^{p}, A^{p}\right)$.

Proof. For (a), we suppose $\lambda_{n} \in D^{1 / 2} l^{\infty, r}$ and write $\lambda_{n}=n^{1 / 2-1 / p} \cdot n^{1 / 2} \cdot \mu_{n}$. Theorem B now implies that $\mu_{n}$ is a bounded (and hence compact, by our last theorem) multiplier from $A^{2}$ to $A^{p}$. Trivially, $D^{1 / 2} \in\left(H^{2}, A^{2}\right)$, and Theorem $F$ tells us that $D^{1 / 2-1 / p} \in\left(H^{p}, H^{2}\right)$ for $1 \leq p \leq 2$. The statement follows.

As for (b), Theorem 5(iii) of [19] tells us that $D^{-1 / p} \in\left(A^{p}, H^{p}\right)$ for all $1 \leq p \leq 2$. This and (a) imply (b).

Zeng [43] observed that $T_{(1 / n)} \in \mathcal{K}\left(A^{p}, A^{p}\right)$, for any $p>1$. Our corollary implies, for instance, that $D^{(p-2-\epsilon) / 2 p}$ is in $\mathcal{K}\left(A^{p}, A^{p}\right)$ for all $1 \leq p<2,0<\epsilon$, which improves the result in [43] for that range. This corollary is only one example of the type of compactness results one can get using the compactness of Wojtaszczyk's multipliers. As another example, Theorem 2.12 of [8] gives a sufficient condition for membership in the multiplier class $\left(A^{p}, A^{q}\right)$ (valid for all $p, q>0$ ). When $q<2$, this condition is proved by factorization through $A^{2}$, and so it actually guarantees compactness (and generalizes Corollary 15(b)).

Finally, we briefly examine the injectivity, surjectivity, and spectrum of some multiplier operators.

PROPOSITION 16. A multiplier operator $T_{\lambda}: A^{p} \rightarrow A^{q}$ is injective if and only if $\lambda_{n} \neq 0$ for all $n$. There are no surjective $\left(A^{p}, A^{q}\right)$ multipliers if $p \neq q$.

Proof. The first statement is obvious. Suppose, for the sake of contradiction in the second part, that $\lambda=\left(\lambda_{n}\right)$ is a surjective $\left(A^{p}, A^{q}\right)$ multiplier with $p \neq q$. Clearly $\lambda_{n}$ can never be zero. Letting $1 / \lambda=\left(1 / \lambda_{n}\right)$, surjectivity implies that $T_{1 / \lambda}$ is also a multiplier from $A^{q}$ to $A^{p}$, and the Closed Graph Theorem tells us that it is bounded. By Theorem 14, at least one of $T_{\lambda}, T_{1 / \lambda}$, is compact, and so their composition is compact. But the composition is the identity map, which is obviously non-compact.

PROPOSITION 17. Suppose $1<p<\infty,\left(\lambda_{n}\right) \in B V$, and $\lambda_{n} \neq 0$ for all $n$. Then the following statements are equivalent: 
(a) $T_{\lambda}: A^{p} \rightarrow A^{p}$ is a Banach space isomorphism.

(b) $T_{\lambda}: A^{p} \rightarrow A^{p}$ is onto.

(c) $T_{\lambda}: A^{p} \rightarrow A^{p}$ has closed range.

(d) $\left(\lambda_{n}\right)$ is bounded away from zero.

Consequently, if $1<p<\infty,\left(\lambda_{n}\right) \in B V$, and $\lambda_{n} \neq 0$ for all $n$, then the spectrum of the multiplier operator $T_{\lambda}$ is $\sigma\left(T_{\lambda}\right)=\overline{\left\{\lambda_{n}: n=0,1,2, \ldots\right\}}$. The eigenvalues are precisely the numbers $\lambda_{n}$.

Proof. By the result from [8] mentioned earlier, we have $T_{\lambda} \in \mathcal{L}\left(A^{p}, A^{p}\right)$. By Proposition 16, $T_{\lambda}$ is one-to-one. Also, (a) $\Rightarrow(\mathrm{b}) \Rightarrow$ (c) is trivial, so it suffices to prove (c) $\Rightarrow$ (d) $\Rightarrow$ (a) to complete the equivalence part.

(c) $\Rightarrow(d)$ : The operator is one-to-one, so (as is well-known) the closed range property is equivalent to $\left\|T_{\lambda} f\right\|_{p} \geq c\|f\|_{p}$ for some $c>0$ and for all $f \in A^{p}$. Now choosing the familiar sequence $f_{n}(z)=(n p / 2+1)^{1 / p} z^{n}$ on the unit sphere of $A^{p}$ yields the desired conclusion: $\left|\lambda_{n}\right| \geq c$.

(d) $\Rightarrow$ (a): It suffices to observe that $\left(\lambda_{n}\right) \in B V$ and $\left|\lambda_{n}\right| \geq c>0$ in turn implies $\left(1 / \lambda_{n}\right) \in B V$. As in the previous proposition, we immediately obtain that the inverse of $T_{\lambda}$ is $T_{1 / \lambda}$, which is also a bounded operator. Thus, (a) follows.

It is quite easy to see that all $\lambda_{n}$ are eigenvalues and that there are no others. The statement about the spectrum of $T_{\lambda}$ can be obtained as follows. Without loss of generality, we may consider only the values of $\mu$ which do not coincide with any $\lambda_{n}$, so the multiplier sequence $\lambda_{n}-\mu$ is in $B V$ and has all non-zero members. Now saying that $\mu \notin \sigma\left(T_{\lambda}\right)$ means that the operator $\mu I-T_{\lambda}=T_{\mu-\lambda}$ is not invertible. But this is equivalent to the boundedness from below of the sequence $\left(\mu-\lambda_{n}\right)$, which is true if and only if $\lambda \notin \overline{\left\{\lambda_{n}: n=0,1,2, \ldots\right\}}$.

The reader will easily observe that the following $A^{2}$ analogue hoids: if $\left(\lambda_{n}\right)$ is an arbitrary bounded sequence such that $\lambda_{n} \neq 0$ for all $n$, then we have the same conclusions (that is, we may completely dispense with the $B V$ assumption).

\section{SOME OPEN PROBLEMS}

We end the paper with some questions which remain to be answered in the future.

QUESTION A. Is it possible to fully characterize the multiplier space $\left(H^{1}, A^{1}\right)$ in terms of some known sequence or function spaces?

QUESTION B. Is the inclusion in Corollary \& strict?

QUESTION C. Can our Theorem 5 be improved so as to obtain a precise description of $\left(A^{p}, A^{2}\right)$ for $1<p<2$ ? 
Perhaps for this purpose one would have to define some new suitable sequence or function spaces which would allow for such a condition.

Acknowledgements. The authors would like to thank P. Wojtaszczyk and J.E. McCarthy for their valuable suggestions. Thanks are also due to the referee, whose comments helped improve the exposition. The first author was supported in part by Forbairt. The third author was partially supported by the DGES(IC) grant PB97-0073.

\section{References}

[1] P. Ahern And M. Jevtić, Duality and multipliers for mixed norm spaces, Michigan Math. J. 30 (1983), 53-64.

[2] J.M. Anderson, J. Clunie, And Ch. Pommerenke, On Bloch functions and normal functions, J. reine angew. Math. 270 (1974), 12-37.

[3] J.M. Anderson, Coefficient multipliers and solid spaces, J. Analysis 1 (1993), 13-19.

[4] J.M. ANDerson AND A.L. Shields, Coefficient multipliers of Bloch functions, Trans. Amer. Math. Soc. 224 (1976), 255-265.

[5] S. AxLER, Bergman spaces and their operators. In: Surveys of Some Recent Results in Operator Theory, eds. J.B. Conway and B.B. Morrel, Pitman Research Notes in Mathematics 171 (1988), Longman, Harlow, 1-50.

[6] O. BlAsco, Operators on weighted Bergman spaces $(0<p \leq 1)$ and applications, Duke Math. J. 66 (1992), 443-467.

[7] O. Blasco, Multipliers on spaces of analytic functions, Canad. J. Math. 47 (1995), $44-64$.

[8] S.M. Buckley, P. Koskela, ANd D. Vukotić, Fractional integration, differentiation, and weighted Bergman spaces, to appear in Math. Proc. Cambridge Phil. Soc.

[9] S.M. BuckLEY, Mixed norms and analytic function spaces, to appear in Proc. Royal Irish Acad.

[10] S.M. BUCKLEY, Relative solidity for spaces of holomorphic functions, preprint.

[11] D.M. CAMPBell AND R.J. LEACH, A survey of $H^{p}$ multipliers as related to the classical function theory, Complex Variables Theory Appl. 3 (1984), 85-111.

[12] P.L. Duren, Theory of $H^{p}$ Spaces, Academic Press, New York, 1970.

[13] P.L. Duren, On the multipliers of $H^{p}$ spaces, Proc. Amer. Math. Soc. 22 (1969), $24-27$.

[14] P.L. Duren ANd A.L. Shields, Properties of $H^{p}(0<p<1)$ and its containing Banach space, Trans. Amer. Math. Soc. 141 (1969), 255-262.

[15] P.L. Duren And A.L. Shields, Coefficient multipliers of $H^{p}$ and $B^{p}$ spaces, Pacific J. Math. 32 (1970), 69-78.

[16] P.L. Duren and G.D. TAYlor, Mean growth and coefficients of $H^{p}$ functions, Illinois J. Math. 14 (1970), 419-423.

[17] T.M. Flett, Mean values of power series, Pacific J. Math. 25 (1968), 463-494.

[18] T.M. FLETT, On the rate of growth of mean values of holomorphic and harmonic functions, Proc. London. Math. Soc. 20 (1970), 749-768. 
[19] T.M. FLETT, The dual of an inequality of Hardy and Littlewood and some related inequalities, J. Math. Anal. Appl. 38 (1972), 746-765.

[20] G.H. HaRdy and J.E. LitTlewood, Some properties of fractional integrals. II, Math. Z. 34 (1932), 403-439.

[21] K.E. HARE, Properties and examples of $\left(L^{p}, L^{q}\right)$ multipliers, Indiana Univ. Math. J. 38 (1989), 211-227.

[22] J.H. Heduund, Multipliers of $H^{p}$ spaces, J. Math. Mech. 18 (1969), 1067-1074.

[23] C. Horowitz, Zeros of functions in Bergman spaces, Duke J. Math. 41 (1974), 693710.

[24] M. Jevtí́ AND I. Jovanović, Coefficient multipliers of mixed-norm spaces, Canad. Math. Bull. 36 (1993), 283-285.

[25] C.N. KellogG, An extension of the Hausdorff-Young theorem, Michigan Math. J. 18 (1971), 121-127.

[26] G. KöтнE, Topological vector spaces. II, Springer-Verlag, Berlin, 1979.

[27] J. Lindenstrauss And A. Peeczyński, Contributions to the theory of the classical Banach spaces, J. Funct. Anal. 8 (1971), 225-249.

[28] J.E. LitTlewood AND R.E.A.C. PALEY, Theorems on Fourier series and power series (II), J. London Math. Soc. 42 (1931), 52-89.

[29] Z. Lou, Coefficient multipliers of Bergman spaces $A^{p}$. II, Canad. Math. Bull. 40 (1997), 475-487.

[30] M. Mateljević AND M. Pavlović, $L^{p}$ behaviour of the integral means of analytic functions, Studia Math. 77 (1984), 219-237.

[31] M. Mateljević and M. Pavlović, Multipliers of $H^{p}$ and BMOA, Pacific J. Math. 146 (1990), 71-84.

[32] A. Nakamura, F. Ohya, and H. Watanabe, On some properties of functions in weighted Bergman spaces, Proc. Fac. Sci. Tokai Univ. 15 (1979), 33-44.

[33] M. PAvlović, Mixed norm spaces of analytic and harmonic functions, II, Publ. Inst. Math. Beograd 41 (55) (1987), 97-110.

[34] A. Pietsch, Operator ideals, VEB Deutscher Verlag der Wissenschaften, Berlin 1978.

[35] W. Rudin, Remarks on a theorem of Paley, J. London Math. Soc. 32 (1957), 307-311.

[36] A.L. Shields AND D.L. Williams, Bounded projections, duality, and multipliers in spaces of analytic functions, Trans. Amer. Math. Soc. 162 (1971), 287-302.

[37] W.T. SLEDD, Some results about spaces of analytic functions introduced by Hardy and Littlewood, J. London Math. Soc. 9 (1974), 328-336.

[38] W.T. SLEDd, Some inequalities related to the Hausdorff-Young theorem, Proc. Amer. Math. Soc. 42 (1974), 535-540.

[39] W.T. SLEdd, On multipliers of $H^{p}$ spaces, Indiana Univ. Math. J. 27 (1978), 797-803.

[40] D. Vukotić, On the coefficient multipliers of Bergman spaces, J. London Math. Soc. 50 (1994), 341-348.

[41] P. WojtaszczyK, Banach Spaces for Analysts, Cambridge University Press, Cambridge, 1991.

[42] P. WojtaszczyK, On multipliers into Bergman spaces and Nevanlinna class, Canad. Math. Bull. 33 (1990), 151-161.

[43] X. Zeng, Toeplitz operators on Bergman spaces, Houston J. Math. 18 (1992), 387-407. 
[44] K. ZHU, Operator Theory on Function Spaces, Marcel Dekker Inc., New York, 1990.

[45] A. Zygmund, Trigonometric series, 2nd ed., Cambridge Univ. Press, Cambridge, 1959.

Stephen M. Buckley

Department of Mathematics

National University of Ireland

Maynooth, Co. Kildare

Ireland

sbuckley@maths.may.ie

Dragan Vukotić

Departamento de Matemáticas

Universidad Autónoma de Madrid

28049 Madrid

Spain

dragan.vukotic@uam.es

1991 Mathematics Subject Classification. Primary 47B38. Secondary 30B10, 46E15.
M. S. Ramanujan

Department of Mathematics

The University of Michigan

Ann Arbor, MI 48109-1109

U.S.A.

msram@math.lsa.umich.edu

Submitted: June 14, 1998 